\title{
A Population at Risk: \\ A Metabolic Syndrome Study among African American Students at a Historically Black College and University
}

\author{
By Steffani N. Driggins ${ }^{*} \&$ Kareem R. Muhammad ${ }^{\dagger}$
}

\begin{abstract}
The aim of the study was to obtain information about what African American undergraduate students attending a Historically Black College and University knew about metabolic syndrome and the risks associated with the syndrome. Approval from the Allen University Institutional Review Board was granted to administer an online anonymous questionnaire about metabolic syndrome to undergraduate students at Allen. The questionnaire was administered using the community based participatory research $(C B P R)$ methodology. The sampling criteria consisted primarily of freshman that were enrolled in the Biological Sciences course, Biology I course, Biology II course, or Freshman Seminar course during the spring 2018 semester and the fall 2018 semester. The sample size consisted of 77 students that completed the online questionnaire on metabolic syndrome. The data from the metabolic syndrome questionnaire was analyzed using descriptive statistics, utilizing percentages from a cross-tabulation of individual responses. The results of the study indicated that some students had a family history of diabetes (41\%), high cholesterol (13.5\%), heart attack (15\%), and stroke (13.5\%). Also, $54.1 \%$ identified the types of diabetes, $17.6 \%$ of the students knew the risk factors of obesity, 31\% knew how high cholesterol is detected, 20.9\% knew the symptoms of a heart attack, and $25.7 \%$ knew the symptoms of a stroke. Based on the results from our study, we hypothesize that introducing a multi-layered educational campaign centered around metabolic syndrome at Allen will increase student knowledge of the risk factors and decrease the number of the African American students that are diagnosed with high blood pressure, high blood glucose levels, and high cholesterol levels.
\end{abstract}

Keywords: African American, College students, Metabolic syndrome, Risk Factors, Universities

\section{Background}

Metabolic syndrome (MetS) is an assortment of interrelated metabolic and clinical risk factors (Gaillard 2018). It includes abdominal obesity, dyslipidemia, glucose intolerance, and hypertension which are associated with an increased risk for cardiovascular disease (CVD) and type 2 diabetes (T2DM) (Gaillard 2018). The National Cholesterol Education Program Adult Treatment Panel III (NCEP ATP III) guidelines states that metabolic syndrome is present if three or more of any of the following criteria are met: 1) waist circumference over 40 inches (men) or 35 inches (women); 2) blood pressure over 130/85 $\mathrm{mmHg}$, fasting triglyceride (TG) level over $150 \mathrm{mg} / \mathrm{dl}$; 3) fasting high-density lipoprotein (HDL) cholesterol level less than $40 \mathrm{mg} / \mathrm{dl}$ (men) or $50 \mathrm{mg} / \mathrm{dl}$ (women); and 4) fasting blood sugar over $100 \mathrm{mg} / \mathrm{dl}$ (Grundy et al. 2005). According to the International Diabetes Federation (IDF) guidelines, a person defined as having metabolic syndrome must

\footnotetext{
*Associate Professor, Allen University, USA.

${ }^{\dagger}$ Dean of Business, Education, and Social Sciences, Allen University, USA.
} 
have central obesity and two or more of the following four factors: 1) reduced HDL cholesterol levels: $<40 \mathrm{mg} / \mathrm{dL}(1.03 \mathrm{mmol} / \mathrm{L})$ in males and $<50 \mathrm{mg} / \mathrm{dL}(1.29$ $\mathrm{mmol} / \mathrm{L}$ ) in females, or treatment for lipid abnormality; 2) raised triglycerides: $\geq 150 \mathrm{mg} / \mathrm{dL}(1.7 \mathrm{mmol} / \mathrm{L})$ or treated for lipid abnormality; 3) raised blood pressure levels: systolic blood pressure $\geq 130 \mathrm{mmHg}$ or diastolic blood pressure $\geq 85 \mathrm{mmHg}$ or previously diagnosed with hypertension; and 4) raised fasting plasma glucose concentration $\geq 100 \mathrm{mg} / \mathrm{dL}(5.6 \mathrm{mmol} / \mathrm{L})$ or previously diagnosed with type 2 diabetes (Ford et al. 2008).

Insulin resistance, abdominal obesity and an inactive lifestyle are risk factors for metabolic syndrome (National Heart, Lung and Blood Institute 2019). Lifestyle, gender, ethnicity, socioeconomic status, psychosocial factors and some inflammatory markers are key components in the pathogenesis of metabolic syndrome (Khan et al. 2015). A study has shown that the principal feature of metabolic syndrome is insulin resistance, resulting in hyperglycemia and hyperinsulinemia, which leads to the development of diabetes (Shulman 2000). The same study found that obesity is the most important predisposing factor for insulin resistance (Shulman 2000). Unfortunately, African Americans have a high percentage of being diagnosed with diabetes (National Center for Chronic Disease Prevention and Health Promotion 2017). Also, obesity is found at disproportionately high rates within the black community (National Center for Health Statistics 2017). In addition, African Americans have a higher risk for cardiovascular disease and having a stroke (Benjamin et al. 2019).

Recently, a study was conducted that analyzed data from the National Longitudinal Study of Adolescent to Adult Health. The study proposed that a higher rate of metabolic syndrome will occur among Hispanic adults and African American adults from disadvantaged backgrounds, after completing their college degree. The study suggests that racial disparity in minorities with a college degree may persist if their health is compromised in young adulthood (Gaydosh et al. 2018).

An analysis of a pooled studies report indicated that metabolic syndrome is prevalent among young adults that are between the ages of 18 and 30 (Nolan et al. 2017). A metabolic syndrome study conducted by Huang et al. in 2004 stated that colleges and universities were important settings for the surveillance, prevention, and intervention of becoming overweight and metabolic syndrome. The study was one of the first studies to examine the associations between obesity and components of metabolic syndrome in college students in the United States. Their study indicated that overweight and metabolic dysfunctions constitute a major health threat at the college age. Since 2004, several metabolic syndrome studies have been conducted with college students. The studies indicated a prevalence rate of metabolic syndrome that was between $3.7 \%$ and $15 \%$. Also, the rate for one criterion of metabolic syndrome was between $16 \%$ and $43 \%$ and the rate of two criterion of metabolic syndrome was between 7.4\% and 20.7\% (Huang et al. 2007, Keown 2009, Fernandes and Lofgren 2011, Dalleck and Kjelland 2012, Topé and Rogers 2013, Morrell et al. 2014, Olfert et al. 2018). Currently, only one metabolic syndrome study with African American college students that has been conducted at an Historically Black College and University has been published. Topè \& 
Rogers (2013) conducted their study at Kentucky State University, which is a Historically Black College and University that predominately consists of African American students. According to the NCEP ATP III guidelines, 31.4\% of their students had 1 criterion for metabolic syndrome and $20.7 \%$ had 2 criteria for metabolic syndrome. However, according to the IDF guidelines, $21.3 \%$ of their students had 1 criterion for metabolic syndrome and $17.5 \%$ had 2 criteria of metabolic syndrome. Overall, the prevalence of metabolic syndrome in the total sample population was 12\% (NCEP ATP guidelines) and 9.3\% (IDF guidelines). The researchers concluded that Historically Black Colleges and Universities offer a unique opportunity to monitor and address the risk factors of metabolic syndrome in a predominantly young African American population (Topè and Rogers 2013).

\section{Aim of Study}

There is a significant lack of research that has been conducted in regards to metabolic syndrome among African American college students that attend a Historically Black College and University. The aim of our study was to obtain information about what African American undergraduate students at Allen University knew about metabolic syndrome and the risks associated with metabolic syndrome.

\section{Methods}

\section{Ethical Approval}

An anonymous online questionnaire on metabolic syndrome was reviewed and approved by the Allen University Institutional Review Board. The questionnaire template in Google Forms was used to create questions pertaining to the following: ethical background, age, gender, classification, major, family health history, individual health and health risks. The second section of the questionnaire assessed the participant's knowledge of the health risks associated with metabolic syndrome.

\section{Sampling Criteria and Size of Participants}

The students that were recruited to complete the metabolic syndrome questionnaire were undergraduate students that were primarily freshman and African American. The community based participatory research (CBPR) methodology (Wallerstein and Duran 2006) was used to conduct the online questionnaire among the undergraduate students at Allen. The participants that participated in the study were enrolled in the Biological Sciences course, Biology I course, Biology II course, or Freshman Seminar course. The students were invited to participate in completing the questionnaire which was administered in the 
spring of 2018 and the fall of 2018 during their class time. These courses were chosen because they consist of large class sizes in comparison to the upper level courses. The students were informed that their participation in the questionnaire was voluntary and that a written consent was required before they could complete the questionnaire. All participants were also informed that they could withdraw from completing the questionnaire at any time without any adverse consequences. Each participant that completed the questionnaire received extra credit points. The consent form that was completed by the participants was maintained in a designated locked facility. A total of 77 participants completed the questionnaire. The demographics of the participants consisted of 97.3\% African American, 4.1\% African, and $1.4 \%$ other. Also, $85.1 \%$ were freshman and $13.5 \%$ were sophomores. A total of $37.8 \%$ of the participants were female and $62.2 \%$ were male. The age of the participants consisted of $4.1 \%$ that were 18 years or younger and $78.4 \%$ that were between 18 and 20 years old. The majority of the participants were business majors $(44.7 \%)$. Also, $28.4 \%$ of the participants were biology majors $(23 \%)$ and $17.7 \%$ were social science majors.

\section{Data Analysis}

The data obtained from the online metabolic syndrome questionnaire was reviewed from the responses recorded on the survey that was created using Google Forms. It was analyzed using descriptive statistics, utilizing percentages from a cross-tabulation of individual responses.

\section{Results}

Among the 77 participants that completed the metabolic syndrome questionnaire, $32.9 \%$ of them exercised every day, $81.1 \%$ identified as being nontobacco smokers, $38.4 \%$ claimed to never have consumed alcohol, $61.1 \%$ claimed to never have used illegal drugs, and $56.8 \%$ did not take prescription medication or get treatment prescribed by a doctor to maintain their health. The personal health information of the participants indicated that $43.6 \%$ had allergies, $25.5 \%$ had asthma, 3.6\% had diabetes, 3.6\% had sickle cell anemia and 1\% had epilepsy. Also, only $46 \%$ of the female participants indicated that they exercised routinely in comparison to $75 \%$ of the male participants.

A total of $33.8 \%$ of the students knew how obesity was defined and $17.6 \%$ knew the risk factors for obesity. In regards to knowledge related to high blood pressure and high cholesterol, just 23\% knew how high blood pressure is measured and $31 \%$ knew how high cholesterol is detected. Also, 20.9\% knew the symptoms of a heart attack, 35.1\% knew when a stroke occurred, and a mere $25.7 \%$ knew about the symptoms of a stroke. Over half of the sample was able to properly identify the risk factors for high blood pressure. The participants also had high percentages associated with their knowledge of diabetes, with $60.8 \%$ of them knowing how diabetes is defined and $54.1 \%$ of them being able to identify the types of diabetes. 
Figure 1. The Family History of Diseases of the Participants that Completed the Metabolic Syndrome Questionnaire

\section{FAMILY HISTORY OF DISEASES OF PARTICIPANTS}

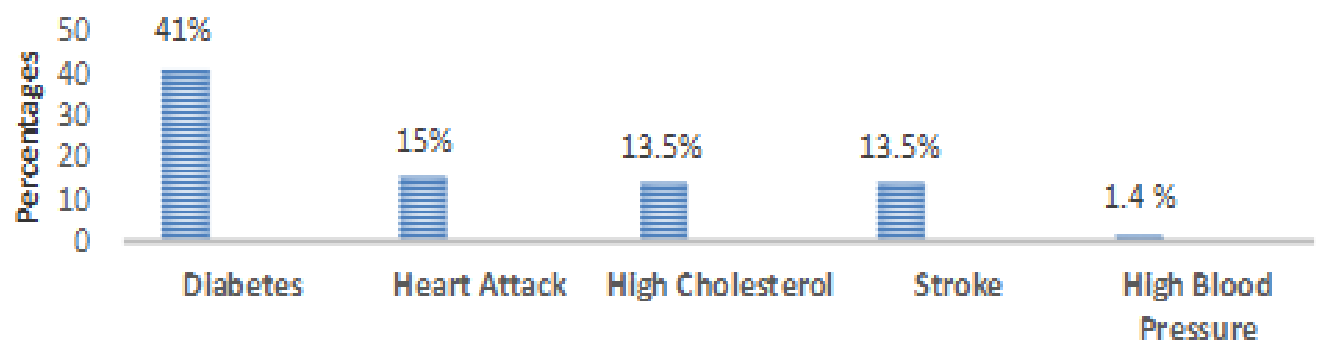

\section{Diseases}

Note: A total of $13.5 \%$ of the participants also indicated that they had a family history of diabetes, heart attack, high cholesterol, stroke and high blood pressure.

Table 1. Female Responses versus Male Reponses on the MetS Questionnaire

\begin{tabular}{|l|l|c|c|}
\hline \multirow{2}{*}{ Conditions } & \multicolumn{1}{|c|}{ Question } & $\begin{array}{c}\text { Females } \\
(\mathbf{n = 3 7 . 8 \%})\end{array}$ & $\begin{array}{c}\text { Males } \\
(\mathbf{n = 6 2 . 2 \%})\end{array}$ \\
\hline \multirow{2}{*}{ Obesity } & How obesity is defined & $38 \%$ & $32 \%$ \\
\cline { 2 - 4 } & Risk factors of obesity & $33 \%$ & $9 \%$ \\
\hline \multirow{2}{*}{ Diabetes } & How diabetes is defined & $75 \%$ & $50 \%$ \\
\cline { 2 - 4 } & Symptoms of diabetes & $67 \%$ & $82 \%$ \\
\hline \multirow{2}{*}{ High Blood Pressure } & Normal blood pressure reading & $1.2 \%$ & $22.5 \%$ \\
\cline { 2 - 4 } & Risk factors of high blood pressure & $63 \%$ & $47.5 \%$ \\
\hline \multirow{2}{*}{ High Cholesterol } & Cause of high cholesterol & $8.3 \%$ & $29 \%$ \\
\cline { 2 - 4 } & Detection of high cholesterol & $17 \%$ & $27.5 \%$ \\
\hline \multirow{2}{*}{ Heart Attack } & Knowing when a heart attack occurs & $42 \%$ & $45 \%$ \\
\cline { 2 - 4 } & Symptoms of a heart attack occurs & $20.8 \%$ & $20 \%$ \\
\hline \multirow{2}{*}{ Stroke } & Knowledge of when a stoke occurs & $37.5 \%$ & $37.5 \%$ \\
\cline { 2 - 4 } & Symptoms of a stroke & $16.7 \%$ & $43.5 \%$ \\
\hline
\end{tabular}

Among the $41 \%$ of participants that had a family history of diabetes, $79 \%$ of them were able to properly identify how diabetes is defined and $67 \%$ of them properly identified all of the symptoms associated with diabetes that were outlined in our questionnaire, including frequent thirst and urination, fatigue and excessive hunger. Of the $13.5 \%$ of participants with a family history of strokes, none of them knew how to detect when a stroke has occurred or the symptoms of a stroke. As of the $15 \%$ participants that had a family history of a heart attack, all of them were able to indicate what happens when a heart attack occurs. However, none of them were able to determine the symptoms of a heart attack. 
Table 2. Responses from Participants with a Family History of Diseases and Responses from Participants without a Family History of Diseases

\begin{tabular}{|l|l|c|c|}
\hline \multirow{2}{*}{ Conditions } & \multicolumn{1}{|c|}{ Question } & $\begin{array}{c}\text { No History of } \\
\text { Disease }\end{array}$ & $\begin{array}{c}\text { History of } \\
\text { Disease }\end{array}$ \\
\hline \multirow{2}{*}{ Obesity } & How obesity is defined & $40 \%$ & $24 \%$ \\
\cline { 2 - 4 } & Risk factors of obesity & $24 \%$ & $9 \%$ \\
\hline \multirow{2}{*}{ Diabetes } & How diabetes is defined & $87 \%$ & $23 \%$ \\
\cline { 2 - 4 } & Symptoms of diabetes & $72 \%$ & $3 \%$ \\
\hline \multirow{2}{*}{ High Blood Pressure } & Normal blood pressure levels & $18 \%$ & $22 \%$ \\
\cline { 2 - 4 } & Risk factors of high blood pressure & $57 \%$ & $45 \%$ \\
\hline \multirow{2}{*}{ High Cholesterol } & Cause of high cholesterol & $19 \%$ & $23 \%$ \\
\cline { 2 - 4 } & Detection of high cholesterol & $18 \%$ & $27.3 \%$ \\
\hline \multirow{2}{*}{ Heart Attack } & Knowing when a heart attack & $37 \%$ & $45 \%$ \\
\cline { 2 - 4 } & Symptoms of a heart attack & $37 \%$ & $18 \%$ \\
\hline \multirow{2}{*}{ Stroke } & Knowledge of when a stoke occurs & $37 \%$ & $45 \%$ \\
\cline { 2 - 4 } & Symptoms of a stroke & $27.2 \%$ & $29.2 \%$ \\
\hline
\end{tabular}

A total of $62 \%$ of the participants indicated a family history of diabetes, heart attack, high cholesterol blood pressure or stroke. A total of $35.1 \%$ of the participants indicated that they did not have a family history of diabetes, heart attack, high cholesterol blood pressure or stroke.

In spite of a familiarity with many of the health risks related to metabolic syndrome, the participants in this study possessed little knowledge on how these afflictions were tested. Overall, $25.7 \%$ of the participants knew the main test used to detect diabetes. A total of $31 \%$ of those with an at-risk family history knew the main test for diabetes and only $14 \%$ of participants without a family history of disease knew the main test used to indicate if someone has diabetes. As stated earlier, $31.1 \%$ of the participants knew the test that was used to detect high cholesterol. This was significantly less that the participants with a family history of diabetes and high cholesterol (44\%).

The students, across multiple categories, purported to exercise at regular rates. A total of $32.9 \%$ of the participants exercised every day, $32.9 \%$ of participants exercised a few times a week, and $19.2 \%$ that exercised a few times a month. A total of $57 \%$ of the participants who were at risk for metabolic syndrome due to their family history exercised regularly and $77 \%$ without such a family history exercised regularly.

Less promising were the results that pertained to the appropriate preventive measures to protect against the risk factors associated with metabolic syndrome. A total of $36.5 \%$ of the participants were able to identify healthy eating as a preventative measure. However, only $12.2 \%$ identified exercising on a regular basis as a preventative measure. Also, only $9.5 \%$ were able to identify maintaining a healthy weight as a preventative measure. Among the participants with a family history of metabolic syndrome risk factors, $31 \%$ were unable to properly identify a preventive strategy. This was also true for an astonishing $59 \%$ of those with no family history of disease. 


\section{Discussion}

The number of participants that completed the questionnaire for our study was low and consisted of a total of 77 participants. This was due to the fact that this is the first phase of an ongoing multi-prong longitudinal study. The second phase of our metabolic syndrome study will consist of administering the questionnaire in the fall of 2019 and the spring of 2020. The same courses will be used to obtain volunteers to participate in the study. The data for both academic years will be used as the foundation of the third phase of the study.

Out of the 77 participants that completed the questionnaire, $43.6 \%$ had allergies, $25.5 \%$ had asthma, 3.6\% had diabetes, 3.6\% had sickle cell anemia and $1 \%$ had epilepsy. Also, the family history of diabetes, heart attack, high cholesterol, stroke and high blood pressure of the participants was $41 \%, 15 \%$, $13.5 \%, 13.5 \%$, and $1.4 \%$ respectfully (Figure 1 ).

Across the board, the students who participated in our anonymous online questionnaire indicated low levels of knowledge of metabolic syndrome, its accompanying factors and how best to avoid becoming victims of what authors such as Grundy et al. (2005) have already labeled as a pandemic. The results from our study are in contrast to the data from a metabolic syndrome study with college students that was conducted by Yahia et al. (2014). Their study indicated that more than $80 \%$ of their participants correctly identified symptoms and complications of diabetes, hypertension, arteriosclerosis, myocardial infarction and stroke on an online metabolic syndrome questionnaire. In our study, less than $50 \%$ of the participants knew the following: 1) how obesity is defined; 2) detection of diabetes; 3 ) risk factors of diabetes; 4) how high blood pressure is measure, 5) detection of high cholesterol; 6) symptoms of a heart attack; 7) knowledge of when a stroke has occurred; and 8) symptoms of a stroke. However, more than half of the participants knew how to identify the risk factors for high blood pressure and $54.1 \%$ of the participants knew how to identify the types of diabetes. Even though the percentages were more than $50 \%$, the percentages are still significantly lower than the results from the study conducted by Yahia et al. (2014).

The percentage of participants in the study conducted by Yahia et al. (2014) that had a family history of diabetes was $23 \%$ which was significantly less than the $41 \%$ of the participants in our study. However, the number of participants in their study that had a family history of high blood pressure was $41 \%$ which was significantly higher than the $1.4 \%$ of participants in our study. The number of participants that were non-smokers in the study conducted by Yahia et al. (2014) was $89 \%$, which was very similar to the $81 \%$ of the participants in our study that were non-smokers. Also, the number of current smokers in their study was $6 \%$ which was significantly the same as the $6.8 \%$ in our study. On the other hand, there was a noticeable difference in the number of African American participants in the study conducted by Yahia et al. (2014), which was $10 \%$. This was an extremely low number in comparison to the number of African American participants in our study which was $97.3 \%$.

Yahia et al. (2014) also stated that the percentage of their participants consisted of $72 \%$ females and $28 \%$ males. Conversely, our study consisted of 
$37.8 \%$ participants that were females and $62.2 \%$ that were males. The difference in gender and correct answers in the study conducted by Yahia et al. (2014) indicated that a higher percentage of female participants correctly answered questions about health conditions associated with metabolic syndrome in comparison to the males. As shown in Table 1, the results of our study indicated a higher percentage of female participants correctly answered questions about health conditions associated with metabolic syndrome in comparison to the males as well. A slightly higher percentage of females knew the risk factors of obesity (38\%) in comparison to the male participants, which was $32 \%$. More females knew how diabetes is defined $(75 \%)$ than males, which was $50 \%$. Also, $63 \%$ of the female participants knew the risk factors of high blood pressure compared to $47.5 \%$ of the male participants. A higher percentage of male participants, however, knew the normal blood pressure reading (22.5\%), cause of high cholesterol (29\%), and detection of high cholesterol (27.5\%). This was in comparison to the female participants whose percentages for the same questions were significantly lower at $1.2 \%, 8.3 \%$, and $17 \%$, respectfully. Gender, however, displayed no significant relationship in knowledge of when a heart attack has occurred, symptoms of a heart attack or knowledge of a stroke.

There was not a significant association with the performance of the participants that had a family history of disease in the results of the metabolic syndrome study conducted by Yahia et al. (2014). However, the participants in our study that had a family history of the components of metabolic syndrome were more knowledgeable than the participants without a family history of components of metabolic syndrome (Table 2). Participants with a family history of health conditions associated with metabolic syndrome knew how obesity is defined $(40 \%)$, risk factors of obesity (24\%), how diabetes is defined (87\%), symptoms of diabetes $(72 \%)$, risk factors of high blood pressure $(57 \%)$, and symptoms of a heart attack (37\%). The responses from the participants that didn't have a family history of the conditions of metabolic syndrome for the same questions were $24 \%$, $9 \%, 23 \%$, and $3 \%, 45 \%$, and $18 \%$ respectively. Overall, both groups of participants would benefit from more knowledge associated with those health conditions.

It was encouraging to see that $79 \%$ of participants with a diabetic family history $(41 \%)$ were able to properly identify how diabetes is defined. It was also heartening to see that $67 \%$ of participants with a family history of diabetes were able to identify all of the symptoms associated with diabetes. However, the $13.5 \%$ participants with a family history of strokes were unable to determine how to determine when a stroke has occurred or the symptoms of a stroke. Also, of the $15 \%$ of participants that had a family history of a heart attack, none of them could determine the symptoms of a heart attack but they all were able to determine what happens when a heart attack occurs. This data is startling because two different metabolic research studies indicated that a family history of a component or components of metabolic syndrome influences the likelihood that the offspring will develop the same health condition(s). Liese et al. (1997) stated that the development of diabetes in the experimental group of their study increased the odds of developing metabolic syndrome if both parents had diabetes and high cholesterol. Santos et al. (2013) indicated in their study that components of 
metabolic syndrome was significantly heritable in their sample of participants and exhibited strong familiar resemblance with correlations between biological relatives of similar magnitude. Thus, given the patterns of family health history unearthed in our survey, our data suggests that shared knowledge of metabolic syndrome would be very beneficial to this group of students. The shared knowledge of metabolic syndrome could possibly reduce their chance of being diagnosed with diabetes, experience a stroke or experience a heart attack, respectively.

The rate of exercise overall was one of the more positive patterns produced from the metabolic syndrome questionnaire data. A total of $32.9 \%$ of the participants exercised daily, $32.9 \%$ of the participants exercised a few times a week, and $19.2 \%$ exercised a few times a month. Also, $57 \%$ of those who were put at risk for metabolic syndrome due to their family history exercised regularly and $77 \%$ of participants without such a family history exercised regularly. The high percentage of participants that exercised daily or a few times a week was due to the fact that a total of $53.8 \%$ of the participants were student athletes in comparison to $46.2 \%$ that were not student athletes. Research has shown that exercise is one of the more effective ways to combat metabolic syndrome. A study conducted by Ford et al. (2005) in regards to sedentary behavior, physical activity, and metabolic syndrome indicated that the participants that did not incorporate moderate of vigorous physical activity during their leisure time had almost twice the chance of having metabolic syndrome. Thus, knowledge of the importance of exercising regularly to reduce the possibility of developing the risk factors of metabolic syndrome or developing metabolic syndrome would be advantageous to the participants that have a family history of a risk factor or risk factors of metabolic syndrome.

In our study, $36.5 \%$ of the participants were able to identify healthy eating as a preventative measure against the risk factors of metabolic syndrome. Also, $12.2 \%$ identified exercising regularly as a preventative measure and only $9.5 \%$ identified maintaining a healthy weight as a preventative measure. This is alarming because less than $50 \%$ of the students were able to correctly identify the proper preventative measures against the risk factors associated with metabolic syndrome. According to Daskalopoulou et al. (2004), one of the treatments used for metabolic syndrome is lifestyle modifications. These modifications include weight reduction and physical activity. Weight reduction, physical activity, and healthy eating are preventative measurements against being diagnosed with any of the risk factors of metabolic syndrome. The fact that less than $50 \%$ of the participants in our study were unable to properly identify one, two or all three of the preventative measures against developing metabolic syndrome indicates how this group would benefit from knowing this information.

\section{Conclusion}

After analyzing the data from our metabolic syndrome study, it is quite apparent that it is imperative to improve the knowledge that our students have about the risk factors of metabolic syndrome by educating them. Just as Yahia et 
al. (2014) concluded, raising our student's awareness about metabolic syndrome based on their pre-existing knowledge of it will enhance their health and wellness. This is especially important since there was a high percentage of students that had a family history of diabetes, high cholesterol, high blood pressure, heart attack and stroke. This is disturbing but it reflects the statistics associated with these health conditions as it relates to the high rates of African Americans being diagnosed with diabetes, obesity cardiovascular disease and stroke (National Center for Chronic Disease Prevention and Health Promotion 2017, National Center for Health Statistics 2017, Benjamin et al. 2019).

Unfortunately, the majority of the studies with college students have been conducted at colleges and universities that predominantly consist of Caucasian males and Caucasian females. It is important to state that our study is the second published study on metabolic syndrome with college students that were predominately African American at a Historically Black College and University. The first study that involved African American college students at a Historically Black College and University was published by Tope et al. in 2013. The study was conducted at Kentucky State University and was a cross sectional study on metabolic syndrome that studied the prevalence rates of criteria associated with metabolic syndrome. The study involved obtaining anthropometrics and biochemical and clinical assessments from each participant. Their study indicated that several participants of their cross-sectional study had 1 criterion $(20.7 \%$, NCEP ATP III guidelines; 31.4\% IDF guidelines) or 2 criteria of metabolic syndrome (20.7\% NCEP ATP III guidelines; 17.5\% IDF guidelines).

Based on our study and the cross-sectional metabolic syndrome study that was conducted by Topè and Rogers (2013), it is obvious that there is a critical need for Historically Black College and Universities and other minority serving institutions, to implement health screenings and educational initiatives for their students. Also, according to the study conducted by Gaydosh et al. (2018), a higher rate of metabolic syndrome will occur among African American adults from disadvantaged backgrounds even if they obtain a college degree. Thus, the authors of this metabolic syndrome study will be collecting more data during the 2019-2020 academic year during the second phase of our study. We will then use the data for both academic years for the third phase of our study. This will involve collaborating with the campus health personnel and local health organizations to introduce a multi-layered educational campus campaign at Allen that is centered around increasing the knowledge of metabolic risk factors among the undergraduate African American students at Allen University. Furthermore, the educational campus campaign will help to decrease the number of students that are diagnosed with diabetes, high blood pressure, high blood glucose levels, and high cholesterol levels. Overall, the program will help to reduce the number of the undergraduate African American students at Allen from being diagnosed with metabolic syndrome as older adults. 


\section{References}

Benjamin E, Muntner P, Alonso A, Bittencourt MS, Callaway CW, Carson AP, et al. (2019) Heart disease and stroke statistics - 2019 update: a report from the American Heart Association. Circulation 139(10): e56-e528.

Dalleck LC, Kjelland EM (2012) The prevalence of metabolic syndrome and metabolic syndrome risk factors in college-aged students. American Journal of Health Promotion 27(1): 37-42.

Daskalopoulou SS, Mikhailidis DP, Elisaf M (2004) Prevention and treatment of the metabolic syndrome. Anthology 55(6): 589-612.

Fernandes J, Lofgren IE (2011) Prevalence of metabolic syndrome and individual criteria in college students. Journal of American College Health 59(4): 313-321.

Ford ES, Kohl HW, Mokdad AH, Ajani UA (2005) Sedentary behavior, physical activity, and the metabolic syndrome among US adults. Obesity Research 13(3): 608-614.

Ford ES, Li C, Zhao G, Pearson WS, Mokdad AH (2008) Prevalence of the metabolic syndrome among US adolescents using the definition from the International Diabetes Federation. Diabetes Care 31(3): 587-589.

Gaillard TR (2018) The metabolic syndrome and its components in African-American women: emerging trends and implications. Frontiers in Endocrinology 8: 383.

Gaydosh L, Schorpp KM, Chenc E, Miller GE, Harrisa KM (2018) College completion predicts lower depression but higher metabolic syndrome among disadvantaged minorities in young adulthood. Proceedings of the National Academy of Sciences 115(1): 109-114.

Grundy SM, Cleeman JI, Daniels SR, Donato KA, Eckel RH, Franklin BA, Spertus JA (2005) Diagnosis and management of the metabolic syndrome. Circulation 112(17): 2735-2752.

Huang TTK, Kempf AM, Strother ML, Li C, Lee RE, Harris KJ, Kaur H (2004) Overweight and components of metabolic syndrome in college students. Diabetes Care 27(12): 3000-3001.

Huang TTK, Shimel A, Lee RE, Delancey W, Strother ML (2007) Metabolic risks among college students: prevalence and gender differences. Metabolic Syndrome and Related Disorders 5(4): 365-372.

Keown TL, Smith CB, Harris MS (2009) Metabolic syndrome among college students. The Journal for Nurse Practitioners 5(10): 754-759.

Khan RJ, Gebreab SY, Sims M, Riestra P, Xu R, Davis SK (2015) Prevalence, associated factors and heritabilities of metabolic syndrome and its individual components in African Americans: the Jackson Heart Study. BMJ Open 5(10): e008675

Liese AD, Mayer-Davis EJ, Tyroler HA, Davis CE, Keil U, Schmidt MI, et al. (1997) Familial components of the multiple metabolic syndrome: the ARIC study. Diabetologia 40(8): 963-970.

Morrell JS, Byrd-Bredbenner C, Quick V, Olfert M, Dent A, Carey GB (2014) Metabolic syndrome: comparison of prevalence in young adults at 3 land-grant universities. Journal of American College Health 62(1): 1-9.

National Center for Chronic Disease Prevention and Health Promotion (2017) National Diabetes Statistics Report, 2017: Estimates of Diabetes and Its Burden in the United States. Retrieved from https://bit.ly/2O6iFJE.

National Center for Health Statistics (2017) Health, United States, 2016: With Chartbook on Long-term. Hyattsville, MD: DHHS Publications.

National Heart, Lung and Blood Institute (2019) Metabolic Syndrome. Retrieved from https://www.nhlbi.nih.gov/health-topics/metabolic-syndrome. 
Nolan PB, Carrick-Ranson G, Stinear JW, Reading SA, Dalleck LC (2017) Prevalence of metabolic syndrome and metabolic syndrome components in young adults: a pooled analysis. Preventive Medicine Reports 7: 211-215.

Olfert MD, Dent A, Wattick RA (2018) Metabolic syndrome prevalence in students attending West Virginia University. Journal of Clinical Medicine 7(12): 487.

Santos DM, Katzmarzyk PT, Trégouet DA, Gomes TN, Santos FK, Maia JA (2013) Familial aggregation of metabolic syndrome indicators in Portuguese families. BioMed Research International 2013(12): 314823.

Shulman GI (2000) Cellular mechanisms of insulin resistance. Journal of Clinical Investigation 106(2): 171-176.

Topè AM, Rogers PF (2013) Metabolic syndrome among students attending a historically black college: prevalence and gender differences. Diabetology and Metabolic Syndrome 5(1): 2.

Yahia N, Brown C, Rapley M, Chung M (2014) Assessment of college students' awareness and knowledge about conditions relevant to metabolic syndrome. Diabetology and Metabolic Syndrome 6(11): 111.

Wallerstein NB, Duran B (2006) Using community-based participatory research to address health disparities. Health Promotion Practice 7(3): 312-323. 